Revue de l'Institut des langues et cultures

d'Europe, Amérique, Afrique, Asie et Australie

37 | 2019

Des genres en Méditerranée : pratiques,

représentations et transfert

\title{
La Méditerranée ou la mer Blanche : couleurs géographiques et noms de mer
}

The Mediterranean Sea or the White Sea?: Geographical Colours and Sea Names

Irène Tamba

\section{OpenEdition}

Journals

Édition électronique

URL : http://journals.openedition.org/ilcea/8313

DOI : 10.4000/ilcea.8313

ISSN : 2101-0609

Éditeur

UGA Éditions/Université Grenoble Alpes

Édition imprimée

ISBN : 978-2-37747-099-0

ISSN : 1639-6073

Référence électronique

Irène Tamba, «La Méditerranée ou la mer Blanche : couleurs géographiques et noms de mer », ILCEA

[En ligne], 37 | 2019, mis en ligne le 03 novembre 2019, consulté le 15 janvier 2020. URL : http://

journals.openedition.org/ilcea/8313; DOI : 10.4000/ilcea.8313

Ce document a été généré automatiquement le 15 janvier 2020

(c) ILCEA 


\section{La Méditerranée ou la mer Blanche : couleurs géographiques et noms de mer}

The Mediterranean Sea or the White Sea?: Geographical Colours and Sea Names

Irène Tamba

\section{Introduction}

1 Le thème à dominante sensorielle, genres, goûts, odeurs et couleurs, retenu pour la $5 \mathrm{e}$ édition du congrès Langues, cultures et médias en Méditerranée m'a remis en mémoire un emploi particulier de la couleur dans le nom turc de la Méditerranée, akdeniz ou «mer Blanche ». En cherchant d'où venait cette dénomination, je suis tombée sur un article datant de 1924, intitulé : "L'origine des noms de mer Rouge, mer Blanche et mer Noire ». L'auteur, Léopold de Saussure (1866-1925), n'était autre que l'un des deux frères $\mathrm{du}$ célèbre linguiste Ferdinand de Saussure. L'explication originale que L. de Saussure propose de cette appellation turque de la Méditerranée m'incite aujourd'hui à reprendre la problématique des couleurs sous l'angle particulier de la dénomination des mers au moyen d'un terme de couleur.

Dans un premier temps, j'exposerai brièvement la thèse de Saussure et les arguments qui plaident pour ou contre le principe explicatif proposé. Selon lui, le nom turc de mer Blanche trouve son origine dans la correspondance entre le blanc et l'ouest dans la cosmogonie chinoise antique, tout comme les autres noms de mer composés des couleurs noire, rouge et jaune. Puis, j'examinerai ces quatre noms colorés des mers dans le cadre de deux autres systèmes de dénomination des espaces marins qui ne relient pas les couleurs et les orientations cardinales: celui de la tradition grécoromaine tout d'abord; puis celui de la nomenclature maritime internationale actuelle. Pour conclure, je reviendrai sur les fonctions descriptive, symbolique et géographique qui caractérisent la sémantique des couleurs. 


\section{La thèse de Saussure (1924) : source chinoise du nom turc de mer Blanche}

3 Léopold de Saussure, sinologue, mathématicien de formation ${ }^{1}$, s'est consacré à l'étude de l'astronomie de la Chine à partir des textes canoniques. Rien d'étonnant dès lors à ce qu'il ait songé au rôle symbolique des couleurs dans la cosmologie chinoise, pour expliquer pourquoi les Turcs installés en Anatolie ont appelé la Méditerranée Akdeniz «mer Blanche » (lit. $A k=$ blanc + deniz = mer) en l'opposant à karadeniz «mer Noire » (lit. Kara $=$ noir + deniz $=$ mer).

4 L'idée de L. de Saussure est à la fois simple et ingénieuse. Selon lui, la plupart des peuples voisins de l'empire chinois, tributaires ou nomades «barbares» - tels notamment les Turcs ou les Mongols - ont adopté la cosmologie de la Chine antique " comme donnant une claire explication des lois de l'univers céleste et terrestre " (1924: 27). Quand, au cours des siècles suivants, certains groupes turcs partis vers l'Ouest se sont installés en Anatolie, ils ont «emporté avec eux les principes de l'antique cosmologie chinoise ». Dans ce système de correspondances symboliques une région à orientation cardinale était liée à une couleur, à un climat, à une saison, à un élément, à une partie du corps, à une planète, bref à « un lot indivis d'attributs » selon l'heureuse formule de Granet (1934¹/1999: 78). Comme les Chinois distinguaient cinq régions, une centrale et quatre périphériques, chacune avait sa couleur " emblématique ». Le jaune était associé au centre ; le sud, au rouge ; le nord, au noir ; l'est, au vert; et l'ouest, au blanc.

5 Sachant que ce sont les Turcs qui ont baptisé la Méditerranée mer Blanche, L. de Saussure a fait l'hypothèse que seule une considération «d'ordre cosmologique » a pu les conduire à l'identifier comme la « mer de l'ouest » par rapport à la mer Noire, « mer du Nord ». Ce qu'oublie de mentionner L. de Saussure, c'est que dans l'antiquité grécoromaine, la mer du Nord en question s'appelait «Pont Euxin », (grec: Pontos Euxeinos, latin: Pontus Euxinus). Quand a-t-elle échangé ce nom pour celui de mer Noire? Selon L. de Saussure (1924 : 31-32), l'appellation de mer Noire aurait été antérieure à l'arrivée des Turcs en Asie Mineure, au XII siècle :

À mon avis, le nom de mer Noire, que les Turcs osmanlis ont trouvé usité en Anatolie, a évoqué dans leur esprit le sens de mer Boréale et ils l'ont complété en attribuant postérieurement le nom de mer blanche à celle qui les limitait à l'ouest.

À cette première difficulté d'ordre historique s'en ajoute une autre, d'ordre géographique. En effet, sur une carte moderne, on constate que c'est la mer Égée qui borde le littoral occidental de la péninsule Anatolienne. L. de Saussure semble donc désigner par le nom de Méditerranée tout le bassin qu'occupe cette mer, sans en distinguer les différentes parties (golfes ou mers côtières). L'appellation générique de mer Méditerranée engloberait donc la mer Égée. Or, l'usage qui s'est imposé aujourd'hui est de dissocier la mer Égée de la Méditerranée, comme l'indique J.P. Pérouse $(1998: 163)$ :

La Méditerranée turque a une extension limitée : elle doit être distinguée de la mer Égée et de la mer de Marmara. Les manuels turcs de géographie décrivent donc une côte méditerranéenne de $1577 \mathrm{~km}$ sur les $8333 \mathrm{~km}$ de côte que compte le pays.

Quelle étendue maritime les Turcs d'Anatolie ont-ils baptisé mer Blanche? Pour répondre à cette question, il faudrait être en mesure de déterminer la position et les limites de la Méditerranée turque autour des $\mathrm{XI}^{\mathrm{e}}-\mathrm{XII}^{\mathrm{e}}$ siècles. Mais la documentation 
actuelle ne le permet pas. Aussi tenterai-je d'explorer une autre voie : celle qu'ouvre la comparaison des systèmes de correspondance entre couleurs et points cardinaux dans les traditions chinoise et turque.

\section{Noms de mer et couleurs des points cardinaux : systèmes turcs et chinois}

8 Aujourd'hui les spécialistes de la Turquie considèrent que les noms de mer Blanche et de mer Noire n'ont rien de surprenant. "On sait en effet, que dans les langues turques anciennes les points cardinaux étaient désignés par des couleurs » (Pérouse, 1998 : 163). La mer qui baigne la côte nord de l'Anatolie est donc la mer Noire, le noir étant la couleur du nord. Quant à la mer Blanche, elle borde «le flanc sud de l'Anatolie », le blanc étant la couleur du sud. Si l'on retrouve ici le principe explicatif qui consiste à associer un point cardinal à une couleur déterminée, on remarque toutefois que le système turc de correspondance entre orientations cardinales et couleurs ne coïncide pas avec celui que L. de Saussure fait remonter à la cosmologie chinoise. Certes, le noir est la couleur du nord à la fois pour les Chinois et pour les Turcs. Mais ces derniers associent le sud au blanc alors que les Chinois considèrent le blanc comme la couleur propre à l'ouest.

9 En adoptant le système de correspondance des couleurs et des points cardinaux de la Chine ancienne, L. de Saussure explique le nom de la mer Blanche qu'il situe à l'ouest de l'Anatolie. Il ne mentionne pas la mer Égée qu'il semble inclure dans la Méditerranée. Pérouse (1998), au contraire, distingue la mer Égée de la Méditerranée. Conformément aux «manuels turcs de géographie» d'aujourd'hui, il décrit la mer Blanche comme ayant une " extension limitée » à la côte méditerranéenne sud. De son côté également, L.-J. Calvet (2015: 25) ne tient compte que des «deux mers» qui bordent la côte septentrionale et méridionale de l'Anatolie :

Lorsqu'ils arrivent au xi siècle en Anatolie les Turcs vont donc désigner les deux mers qui la bordent selon leurs points cardinaux, d'où Karadeniz (m.à.m. «mer noire ») mais en fait mer du Nord et Akdeniz ( «mer Blanche » c'est-à-dire du Sud).

Cette comparaison montre que L. de Saussure et L.-J Calvet découpent et positionnent différemment l'espace maritime qu'ils appellent mer Blanche. Par ailleurs, Calvet, à la suite des turcologues, s'intéresse au couple formé par les deux mers et par les deux couleurs, kara «noir » et ak "blanc », alors que Saussure se focalise sur la localisation géographique des mers, appariant la mer du Nord à la mer du Sud. Mais, tous deux recourent au même principe explicatif en attribuant une couleur aux directions géographiques cardinales. Ce qui fait apparaître une double divergence au niveau de la signification directionnelle des couleurs. Le blanc est la couleur de l'ouest dans le système chinois mais la couleur du sud dans le système turc. D'autre part, le rouge est la couleur du sud dans la cosmologie chinoise mais de l'Est chez les Turcs.

11 D'où le problème posé par le nom grec Eruthrè thalassa, "mer Érythrée », composé de l'adjectif eruthrè "rouge ", ainsi que son calque latin mare Rubrum. L. de Saussure voit dans ce nom une confirmation de la correspondance entre le rouge et le sud. Et il en trouve une preuve chez Hérodote ( $\mathrm{vI}^{\mathrm{e}}$ siècle avant notre ère), qui parle de «la mer du Sud appelée Érytrée $»^{2}$ (Hist. IV, 37). 
12 Mais qu'en est-il pour les Turcs? Pour eux, c'est l'opposition complémentaire entre noir et blanc qui prévaut. Aussi n'apparient-ils pas la mer Noire à la mer Rouge, en opposant la couleur du nord à celle du sud. Sinon, leur système de correspondance entre sud et blanc conduirait à appeler mer Blanche, la mer Érythrée, située au sud. Or, les Turcs utilisent aujourd'hui kizil deniz («mer rouge ») pour désigner la mer Rouge.

13 L'hypothèse saussurienne d'un emprunt à la cosmologie chinoise du principe associant cinq couleurs aux cinq directions cardinales ne permet donc pas de rendre compte des couleurs intégrées dans les noms de mer turcs, par association aux couleurs désignant les points cardinaux. D'où la nécessité d'examiner de plus près les systèmes de correspondance impliquant des couleurs en chinois et en turc.

\subsection{Symbolisme des couleurs dans la cosmologie chinoise}

L. de Saussure se réfère aux classiques chinois pour mettre au jour la dimension cosmologique des cinq couleurs symboliques, noir, rouge, vert, blanc, jaune. Elles ne sont pas seulement associées au cinq régions cardinales (nord, sud, est, ouest, centre) mais aussi aux cinq éléments ${ }^{3}$, aux cinq saisons, aux cinq activités, aux cinq bonheurs, aux cinq saveurs, etc. Le « souverain œcuménique » siégeait sur son trône qui « était rituellement tourné face au sud ».

Il avait ainsi derrière lui la région du nord, du froid, des ténèbres et de l'humidité, correspondant à l'élément eau et à la couleur noire; devant lui, la région du sud, de la chaleur, de la lumière et de la sécheresse, correspondant à l'élément feu et à la couleur rouge; à sa gauche, la région de l'est, du matin, du printemps, de la végétation, correspondant pour cette raison à l'élément bois et à la couleur verte; à sa droite, la région de l'ouest, du soir, de l'automne, correspondant à l'élément destructeur, le métal, et à la couleur blanche du deuil. Quant à la région centrale, elle correspond comme de juste à l'élément terre dont la couleur est naturellement jaunâtre. C'est pourquoi, en Chine, la couleur impériale, celle du centre et du souverain seigneur de toute la terre, est le jaune. (Saussure $1924: 25$ )

Comme on peut le constater, les couleurs et les orientations cosmiques s'inscrivent dans un système global d'organisation de l'univers, auquel elles doivent leur durée et leur invariance. Qu'en est-il de la correspondance entre les couleurs et les noms de mers dans le cas turc?

\subsection{Couleurs et points cardinaux dans la géographie turque}

16 Pour les Turcs, nous l'avons vu, l'existence d'un principe de corrélation entre un point cardinal et une couleur ne fait aucun doute. En tant que linguiste, Calvet (2015: 25) adopte ce principe et y ajoute une précision d'ordre sémantique. Le nom de couleur fonctionne comme une désignation métonymique des points cardinaux et des deux mers qu'ils servent à localiser :

Le noir (kara) désignait le nord, le rouge l'ouest, le jaune l'est et le blanc ( $a k$ ) le sud.

Un tel mode de dénomination métonymique aurait été utilisé seulement pour (re)baptiser les deux "grandes mers» bordant la péninsule anatolienne: la Méditerranée et la mer Noire sans tenir compte de leurs subdivisions en mers côtières. Calvet, par exemple, passe sous silence la mer Égée. Comme elle baigne la côte ouest de l'Anatolie, direction désignée par le rouge dans le système de dénomination turc des mers, elle aurait dû s'appeler kizil deniz « rouge mer ». Or, le nom kizil deniz n'est pas 
employé à date ancienne pour désigner la Méditerranée. Il a été forgé à l'époque moderne comme calque de traduction de l'actuelle mer Rouge.

Enfin, le principe turc de corrélation entre directions cardinales et couleurs basiques semble s'accommoder de variations historiques. Il diffère par là du système cosmologique chinois invariant qui fixe la correspondance entre cinq couleurs et les cinq orientations canoniques.

Selon D. Cureau, en effet, (Blog, 14 juillet 2017), Wikipedia enregistre quatre systèmes turcs mettant en relation les points cardinaux et les couleurs. Il les présente, dans le tableau suivant, avec leur site de référence :

\begin{tabular}{|c|c|c|c|c|c|c|}
\hline Turquie & $\mathrm{N}$ & E & $\mathbf{s}$ & W & c & Source \\
\hline 1 & & & & & - & https:/fr.wikipedia.org wikiRussie blanche \\
\hline 2 & & - & & - & 一 & https:/fr.wikipedia.org/wikiMer Noire \\
\hline 3 & & & & & - & https://it.wikipedia.org/wikiPunto cardinale \\
\hline 4 & & & & & & https://en.wikipedia.org/wiki Cardinal direction \\
\hline
\end{tabular}

Sur la première ligne, figure le système standard des quatre couleurs des points cardinaux adopté par Calvet (2015:25) :

Kara, le « noir » désigne le nord,

$A k$, le «blanc » désigne le sud,

kızll, le « rouge » désigne l'ouest,

Yeşil, le « vert » ou Sari, le « jaune » désignent l'est.

21 Sur la deuxième ligne, seuls deux points cardinaux sont pris en considération : le nord désigné par ak (blanc comme neige), et le sud par kizil (rouge comme la chaleur). Ces deux couleurs seraient l'apanage des « anciens turcophones de la steppe ».

Les lignes 3 et 4 sont superposables, à la dernière colonne près. La ligne 4 comporte un cinquième point cardinal, le centre, associé non au jaune comme dans le système chinois mais au vert.

Par ailleurs, si l'on tient compte du recouvrement lexical du bleu et du vert, on voit que les quatre premières couleurs des points cardinaux turcs aux lignes 3 et 4 coïncident avec celles du système chinois :

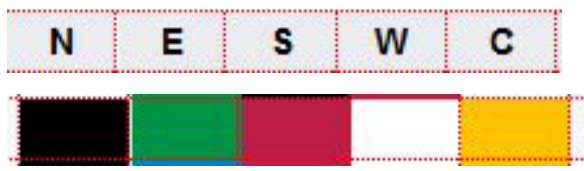

Cette coïncidence serait un argument en faveur de la thèse saussurienne d'un emprunt des Turcs nomades des steppes à leurs voisins chinois. Surtout si l'on se range derrière les turcologues qui soutiennent que "toutes ces appellations pourraient avoir une origine turco-mongole » et que le système actuel serait secondaire :

la logique désignant le nord (obscur) par le noir, le sud (la clarté) par le blanc et l'ouest (soleil couchant) par le rouge, ne serait apparue que tardivement, en Asie mineure 4 . (Cureau, 2017)

On peut tirer une première conclusion positive de la thèse saussurienne. Le recours au principe d'une correspondance symbolique entre un point cardinal et une couleur apporte bien une réponse unifiée à la question de l'origine des couleurs dans les noms des mers Blanche, Noire et Rouge. 
Mais, le principe turc d'une correspondance entre couleurs et points cardinaux qui remonterait à la cosmologie chinoise ou védique ${ }^{5}$ ne peut rendre compte ni du décalage existant entre les systèmes chinois et turcs reliant points cardinaux et couleurs; ni de l'instabilité du symbolisme turc des couleurs cardinales face à la stabilité du symbolisme chinois; ni de l'internationalisation de mer Noire mais de l'abandon, sauf chez les Turcs, de mer Blanche au profit de mer Méditerranée.

D'où l'idée d'examiner l'interprétation des noms de mer composés d'un terme de couleur dans le cadre de systèmes de dénomination, où les couleurs ne sont pas reliées à des points cardinaux. Le premier est celui des noms de mers dans l'antiquité grécoromaine. Le second, la nomenclature unifiée des océans et des mers mise en place par l'Organisation Hydrographique Internationale (OHI) en 1953.

\section{Des noms de mers sans couleur dans la géographie gréco-romaine}

Dans son article sur l'origine des noms de mers composés d'une couleur, L. de Saussure n'accorde aucune attention à la manière dont ces mers étaient nommées par les Grecs et les Romains dans l'antiquité à une exception près : celle de la mer appelée Érythrée ou « rouge ». Et pour cause: il n'existe manifestement pas de lien entre les points cardinaux et les couleurs dans la tradition géographique gréco-latine. Ni l'appellation de mer Noire, ni celle de mer Blanche ne sont attestées dans les documents géographiques de la Grèce antique ou des Romains. Grecs et Romains nommaient l'actuelle mer Noire, Pont Euxin ou Pont. Et le nom de Méditerranée ne s'est imposé que tardivement, sauf en Turquie où l'appellation de mer Blanche (Akdeniz) s'est maintenue. Chaque nom a donc connu une évolution spécifique qui peut aider à mieux comprendre l'absence de relations entre le système gréco-romain de dénomination des mers et les couleurs rouge, noire et blanche des points cardinaux.

\subsection{Mer Érythrée et mer Rouge}

29 À l'appui de sa thèse, L. de Saussure avance une preuve découverte dans les Histoires d'Hérodote. Il s'agit de l'identification explicite de la mer Érythrée à une mer Australe, thalassa notiè (lit. mer " notique ", de notos = vent du sud). En considérant que le nom de mer Rouge remonte étymologiquement à la dénomination grecque de mer Érythrée, Saussure transfère à la mer Rouge la qualification de mer du sud que reçoit la mer Érythrée. Certes, la mer Rouge et la mer Érythrée ne coïncident pas, puisque «notre mer rouge actuelle » n'est qu'un " golfe », kolpos arabios, "golfe Arabique » de la mer Érythrée à l'époque d'Hérodote. Mais, argumente Saussure, «il était naturel que les Grecs, sachant que ses eaux se prolongeaient jusqu'aux rives perses, l'aient englobée dans le nom générique de mer Rouge ou mer Australe » (1924:29).

L'explication saussurienne du «nom de mer Rouge attribué à la mer australe (mer de l'Inde ou océan indien)» (ibidem) ne rend pas compte des différentes mers nordiques auxquelles elle est opposée dans les Histoires. Ainsi n'est-ce pas la même mer du Sud qui est couplée à ses pendants nordiques. En allant d'Est en Ouest, Hérodote oppose la mer boréale fermée qu'est la mer Caspienne (Histoires I, 203) au golfe Persique, ou partie de la mer Érythrée qui baigne le littoral sud de la Perse. Puis, au niveau de l'Asie centrale, 
il énumère les peuples qui se succèdent de la mer du sud ou mer Érythrée à «la mer boréale, où se jette le Phase » (Hist. IV, 37). On dirait aujourd'hui de l'océan Indien à la mer Noire (où se jette le Rion). Enfin, Hérodote imagine que le delta du Nil a dû autrefois former un golfe, comparable et parallèle au golfe Arabique, mais en sens inverse. Ce golfe pénètre dans les terres en direction de l'Éthiopie à partir de la mer boréale c'est-à-dire de la Méditerranée ou "notre mer ", tandis que le golfe Arabique part de l'Érythrée ou mer Australe (Hist. II, 11) ${ }^{6}$ vers la Syrie.

31 Saussure se borne donc à identifier la mer Érythrée à une grande mer australe, qui doit la couleur rouge de son nom à son opposition à la mer boréale, à laquelle elle est appariée de part et d'autre du territoire turc en Asie Mineure. Telle n'est pas l'optique d'Hérodote qui décrit plutôt des golfes ou mers bordières de la côte australe de la mer de l'Inde (notre océan Indien), opposés à des mers nordiques ${ }^{7}$.

Par ailleurs, Saussure adopte l'étymologie qui fait de Érythrée la forme féminine de l'adjectif grec eruthros, « rouge ». Ce sens fonde le rapprochement entre le nom de mer Rouge et la couleur d'une direction cardinale, que ce soit le sud dans la cosmologie chinoise, ou l'ouest pour les géographes turcs. Mais bien que devenue classique, l'étymologie qui rattache Érythrée à l'adjectif signifiant rouge était déjà contestée par les Romains ${ }^{8}$. Ce nom viendrait d'un personnage légendaire éponyme, Erythras. Selon certains, Erythras serait le nom d'un chef local perse, tandis que pour d'autres il s'agirait du gardien d'un des haras du golfe Persique ${ }^{9}$. Et, pour d'autres encore, Erythrès serait un fils du roi Persée, héros de la mythologie grecque qui parvint à vaincre les Gorgones.

Dans le premier cas, le rouge reçoit une interprétation descriptive. Il s'agit d'une couleur temporaire ou locale de la mer. C'est ce qu'attestent les différentes justifications que rapporte Strabon (60 avant J.-C. - 20 après) dans sa Géographie (XVI, 20 ) : réflexion de la lumière solaire à midi ou reflet d'une montagne surchauffée; ou encore, eaux rouges d'une source se déversant dans la mer. Huit siècles plus tard, on retrouve un point de vue similaire chez Isidore de Séville qui attribue le nom de la mer Érythrée à ses "flots rouges, proches de la couleur du sang", en raison de la pigmentation rouge de la terre du littoral. Avec l'érosion, cette terre s'écoulerait dans les rivières et dans la mer (Etym., XIII, 17).

34 Ces diverses explications "naturalistes " sont tributaires de la couleur rouge de leur référent, la mer Érythrée. Or, l'identification de cette mer varie selon les auteurs grécoromains ${ }^{10}$. D'où des justifications qui diffèrent suivant que la mer Érythrée désigne le golfe Persique, le golfe Arabique ou la côte occidentale de l'océan Indien ou encore globalement « la mer de l'Inde et ses golfes».

Dans le second cas, comme la mer Érythrée tire son nom d'un héros éponyme, le problème disparaît avec l'absence de tout rapport à la couleur rouge.

Enfin, Bačić (1995 : 189) évoque une troisième hypothèse plus récente :

Il est largement admis aujourd'hui que les Grecs de l'antiquité ont appris des Égyptiens le nom de mer Rouge. Ceux-ci auraient transféré à la mer adjacente l'attribut de la Terre Rouge, nom qu'ils utilisaient pour désigner le Désert du Sud, par opposition à l'Égypte proprement dite, appelée Terre Noire ${ }^{11}$.

37 Comme on peut le constater, il s'agit ici encore de couleurs géographiques. Mais l'opposition de la Terre rouge, stérile, du désert à la Terre Noire de la plaine fertile du Nil ne met pas directement en cause deux directions cardinales. Si le rouge est 
effectivement associé au désert du Sud, le noir ne correspond à aucune orientation cardinale. aujourd'hui appelée mer Noire. Le nom grec Pontos Euxinos et son calque de traduction en latin, Pontus Euxinus, ne comportent aucune indication intrinsèque de couleur ${ }^{18} \mathrm{ni}$ d'orientation. Comment, alors, les Turcs auraient-ils pu identifier le Pont Euxin à une mer dite Noire parce que située au nord de leur contrée?

Saussure (1924: 31) laisse entendre que les Turcs installés en Anatolie avaient trouvé plusieurs dénominations concurrentes pour désigner la mer Noire. À côté de Pont Euxin, toujours utilisé par les Byzantins à la suite des Romains et des Grecs, apparaissent les appellations de "mer Russe » et de "Bâhr el aswad» (sic). Ce nom arabe peut signifier "mer Noire $»^{19}$ mais aussi «mer Majeure ». Car, précise Saussure, on a observé "que tant en arabe (aswad) qu'en turc (kara), le même mot possède la double signification de "plus grand" et de "noir" $»^{20}$. Ce qui lui permet de rapprocher ces noms de celui, plus tardif, de Mare Maggiore que les marins italiens donneront au XIV ${ }^{e}$ siècle à cette mer. Comment les Turcs d'Anatolie en sont-ils arrivés à interpréter kara deniz comme mer Noire plutôt que comme mer Majeure? Osman Karathay (2011) propose une explication 
qui, nous allons le voir, permet de rendre compte des deux dénominations turques de mer Noire et de mer Blanche face à celles, byzantines, de Pont Euxin et de Méditerranée.

\subsection{Mer Noire et mer Blanche méditerranéenne}

Selon O. Karathay, les Osmanlis en arrivant en Asie mineure ont utilisé, dans le courant du XIV e siècle, le nom de kara deniz emprunté aux Turcs qui habitaient au nord de la mer Noire. Or, chez ces peuples turcs, kara avait perdu son sens médiéval de "grand, puissant ", si bien que kara deniz signifiait «mer Noire ». Sens attesté notamment dans les dénominations Bulgare, Hongroise ou Khazar de la mer Noire, dès le haut Moyen Âge. À leur arrivée en Anatolie, les Turcs, à leur tour, auraient adopté le nom de kara deniz avec le sens de «mer Noire ». Et comme l'Anatolie est bordée par deux mers, la première ayant reçu le nom de mer Noire, la seconde a tout naturellement été appelée mer Blanche ${ }^{21}$. Car le noir est couplé à son opposé complémentaire le blanc, comme la nuit au jour, ou l'ombre à la lumière. Un tel couplage sémantique est indépendant de la localisation septentrionale ou méridionale de ces deux mers, puisque pour les Turcs du Nord la mer Noire se trouve au Sud, alors que pour les Turcs anatoliens, elle se trouve au nord.

En conséquence, si la couleur blanche n'a plus sa valeur de direction cardinale, Saussure et les turcologues sont renvoyés dos à dos: la mer Blanche ne se situe ni à l'ouest ni au sud, mais elle est opposée à la mer Noire ${ }^{22}$, qui borde l'autre côte de l'Anatolie ${ }^{23}$.

Reste à justifier la diffusion de mer Noire et l'élimination de Pont Euxin, alors que la Méditerranée l'emporte sur la mer Blanche, qui ne se maintient qu'en turc. Un facteur historique a dû contribuer à cette double évolution. Le nom de Mer Noire était bien installé au nord de l'Europe, avant que les Turcs d'Anatolie ne l'adoptent. Par contre, le nom de mer Blanche donné par les Turcs à la Méditerranée par opposition à la mer Noire ne pouvait pas être compris par les riverains de la Méditerranée qui considéraient que leur mer était centrale et indépendante de la mer Noire. Aussi est-ce l'appellation plus familière de Méditerranée qui a prévalu sur celle, néologique, des Turcs. Et, aujourd'hui, selon la terminologie géographique internationale, la mer qui porte le nom de mer Blanche est celle qui se situe au sud de la mer de Barents dans l'océan Arctique. Quant à la couleur de son nom, elle n'indique pas une localisation géographique, ou positio loci, mais une propriété physique ou qualitas corporis selon l'opposition de Bačić (1995 : 192).

\section{Couleurs et noms des mers dans la nomenclature géographique actuelle}

Aujourd'hui, il existe une nomenclature géographique internationale unifiée, relativement récente, puisqu'elle remonte aux directives données par une commission intergouvernementale, l'Organisation Hydrographique Internationale (OHI, ou $\mathrm{IHO}$ en anglais). L'OHI a défini un certain nombre de principes et de paramètres qui lui ont permis de dresser un inventaire consensuel d'une soixantaine de noms d'espaces maritimes, associés à des descriptions qui en indiquent la localisation et les limites. Cette nomenclature standard comporte des noms généralement binaires, composés de 
deux éléments dotés d'une fonction distincte : un formant catégorisant $-F c a t-$ et un formant singularisant - Fsing - (cf. Bosredon et Tamba, 2018).

Dans le cas des mers, la liste française de l'OHI (1953) comporte 48 noms composés du Fcat mer et de diverses sortes de déterminants nominaux en fonction de Fsing. Parmi ces derniers, les Fsing de couleur constituent un mini-groupe de quatre noms :
1. mer Rouge
2. mer Noire
3. mer Blanche
4. mer Jaune

Ce groupe minoritaire s'oppose à deux autres groupes plus importants : un premier groupe majoritaire de 41 noms propres de lieux, qui servent à localiser les mers au moyen d'un repère toponymique terrestre (pays, villes, ou habitants des zones côtières); et un second groupe plus réduit de 7 noms propres de personnages historiques, éponymes, qui situent dans le temps la découverte de la mer (Tamba, à paraître $)^{24}$. Une différence essentielle entre les Fsing de couleur et les Fsing toponymes ou éponymes retiendra ici l'attention. Alors que les noms propres de personnes ou de lieux sont transposés phonétiquement dans les différentes langues, les Fsing de couleur, eux, sont régulièrement traduits par des termes de couleur comme les adjectifs en français. Ce qui a pour effet de réactiver leur sens lexical de couleur et d'entraîner une remotivation de ces quatre noms en rapport avec quelque propriété naturelle de ces mers (Tamba, à paraître). Dès lors, toute interprétation d'orientation géographique se trouve éliminée.

Mais, le statut référentiel de la couleur dans ces noms de mers reste paradoxal. On le motive, en effet, par des propriétés physiques qui ne sont pas forcément visibles. Si, en effet, on regarde les photos envoyées par les satellites ou si l'on observe ces mers, on constate que les couleurs de leurs eaux varient avec le temps: bleu/vert par beau temps ou gris/noir en cas de tempête, sous un ciel d'orage; ou encore en fonction de leur profondeur et de la nature de leurs fonds (algues, sables, rochers, coraux)...

Les géographes confirment cette uniformité des couleurs des mers, en adoptant une convention cartographique internationale qui représente en bleu les étendues maritimes par opposition aux couleurs marron et vert réservées aux hauteurs ou plaines terrestres. Comme l'écrit Dainville (2018 : 209) :

Les couleurs dont dispose le géographe sont réduites à un petit nombre [...] mais elles constituent une vraie langue, familière aux auteurs et aux usagers des cartes.

51 Mais l'uniformisation de ces conventions est récente. Les couleurs des cartes ont une longue histoire déterminée à la fois par les techniques de coloration ou de dessin et par la symbolique des couleurs (Dainville, 2018 : 210-214). Par exemple, la mer Rouge est " souvent colorée en vermillon jusqu'au XVIII ${ }^{\mathrm{e}}$ siècle sur de nombreuses cartes portulans décoratives » (Vagnon, 2017 : 139 et suiv., cf. par ex. la reproduction de l'atlas catalan de 1375).

\section{Conclusion}

$52 \mathrm{Au}$ terme de ce périple sémantique à travers les couleurs dans les noms de mer, on retiendra un triple usage des couleurs des mers. Il peut s'agir de couleurs physiques, perceptibles à l'œil humain; de couleurs conventionnelles qui servent aujourd'hui à 
représenter les mers sur les cartes; et, enfin, de couleurs intégrées aux noms des mers à valeur autrefois symbolique mais aujourd'hui descriptive.

\section{BIBLIOGRAPHIE}

ARISTOTE, Météorologiques. Livres I et II (t. 1, 2002) ; Livres III et IV (t. 2, 1982), Paris : Les Belles Lettres.

AUJAC Germaine (1975), La géographie dans le monde antique, PUF : Que sais-je?

AUJAC Germaine (2012), Claude Ptolémée, Astronome, astrologue, géographe (3éd.), Paris : CTHS.

BAČIć Jacques (1995), Red sea - Black Russia, Prolegomena to the History of North Central Eurasia in Antiquity and Middle Ages, New York : East European Monographs, distributed by Columbia University Press.

BOSREDON Bernard (1994), Les titres de tableaux. Une pragmatique de l'identification, Paris : PUF.

BOSREDON Bernard \& TAMBA Irène (1999), « Une ballade en toponymie : de la rue Descartes à la rue de Rennes », Linx (40), 55-69.

BOSREDON Bernard \& TAMBA Irène (2018), « Noms des mers et des océans : une nomenclature géographique interculturelle », communication présentée au $8^{\mathrm{e}}$ colloque international Intercultural Pragmatics and Communication, Université de Chypre.

BRATARIU Georges I. (2009), La mer Noire, des origines à la conquête ottomane, Paris : éditions Kryos.

CALVET Louis-Jean (2016), La Méditerranée : mer de nos langues, Paris : CNRS Éditions.

CHENG Anne (1997), Histoire de la pensée chinoise, Paris : Seuil.

DAINVILLE DE François (2018), Le langage des géographes. Termes, signes, couleurs des cartes anciennes (1500-1800) [1964], Paris : CTHS.

GAUTIER Raoul (1926), « Léopold de Saussure (1866-1925) », Le Globe, Revue genevoise de géographie (65), 25-39.

GRANET Marcel (1934), La pensée chinoise (rééd. en 1999), Paris : Albin Michel.

GORDEEV Anton (2007), Cartography of Black and Azov seas: Retrospective up to 1700, Moscou,en ligne sur https://www.academia.edu/8138553/

Cartography_of_the_Black_Sea_and_the_Sea_of_Azov_Retrospective._Up_to_1700.

Grataloup Christian (2015), «L'invention des océans. Comment l'Europe a découpé et nommé le monde liquide », en ligne sur Géoconfluences : http://geoconfluences.ens-lyon.fr/informationsscientifiques/dossiers-thematiques/oceans-et-mondialisation/articles-scientifiques/l-inventiondes-oceans.

HÉRODOTE, Histoires. Livre I (2010) ; Livre II (2002) ; Livre IV (1949), Paris : Les Belles Lettres.

ISIDORE DE SÉVILLE, Etymologiarum sive Originum, liber XIII, en ligne sur https:// www.thelatinlibrary.com. 
KISH George (1980), La carte, image des civilisations, Paris : Seuil.

MAUSS Marcel \& DURKHEIM Émile (1969), « De quelques formes primitives de classification », M. Mauss (dir.), Essais de sociologie, Paris : Éditions de Minuit, 162-230.

Pelletier Philippe (2005), « Où est passée la mer d'Orient », M. Viegnes (dir.), Imaginaire des points cardinaux, aux quatre angles du monde, Paris : Imago, 285-314.

PÉROUSE Jean-François (1998), « La mer Blanche du point de vue turc ; ou en quoi la Turquie est-elle aussi méditerranéenne? », Hérodote (90), Paris, 163-177.

PLINE L'ANCIEN ( $\mathrm{I}^{\mathrm{er}}$ s.), Histoire naturelle. Livre VI (éd. tr. fr. 2005), Paris : Les Belles lettres.

RouGÉ Jean (1988), « La navigation en mer Érythrée dans l'Antiquité », Jean-François Salles (dir.), L'Arabie et ses mers bordières. 1. Itinéraires et voisinages. Séminaire de recherche 1985-1986, Lyon : Maison de l'Orient et Presses Universitaires de Lyon, 59-74.

SCHNEIDER Pierre (2001), « Hè mégalè thalassa : un autre nom de l'Érythrée ? ", REG, tome 114, 626-636.

SAUSSURE DE Léopold (1924), «L'origine des noms de mer Rouge, mer Blanche et mer Noire », Le Globe. Revue genevoise de géographie, tome LXIII, 23-36.

STRABON, Géographie. Livre I (2018) ; Livre II (1969), Paris : Les Belles Lettres.

SUN-DURAND Chaoying (2005), « La pérégrination vers l'Ouest (Xiyou-ji) et les cinq points cardinaux chinois ", M. Viegnes (dir.), Imaginaire des points cardinaux, aux quatre angles du monde, Paris : Imago, 315-328.

TAMBA Irène (à paraître), « A Geographic and Lexical Puzzle: Colors in Names of Seas », A. Vovin Kanreki Festchrift, John Kupchik ed.

VAGNON Emmanuelle \& VALLET Éric (2017) [dir.], La fabrique de l'océan Indien. Cartes d'Orient et d'Occident (Antiquité - XVI siècle), Paris : Publications de la Sorbonne.

VAGNON Emmanuelle (2017), « Mer Rouge et golfe Persique dans les cartes occidentales », E. Vagnon \& É. Vallet (dir.), La fabrique de l'océan Indien. Cartes d'Orient et d'Occident (Antiquité - XVI siècle), Paris : Publications de la Sorbonne, 139-150.

VAllet Éric (2017), «Les noms de l'Océan » et « Mer Rouge et golfe Persique dans la cartographie, des Grecs aux Arabes », E. Vagnon \& É. Vallet (dir.), La fabrique de l'océan Indien. Cartes d'Orient et d'Occident (Antiquité - XVI ${ }^{e}$ siècle), Paris : Publications de la Sorbonne, 111-124, 125-138.

VIEGNES Michel (2005) [dir.], « Introduction », Imaginaire des points cardinaux, aux quatre angles du monde, Paris : Imago.

\section{NOTES}

1. L. de Saussure, entré à l'école navale de Brest, en sortit, très jeune, officier de la Marine française et, à ce titre, sillonna les mers lointaines. Ayant pris une retraite prématurée vers trente ans, il se consacra à l'étude du chinois et de la tradition astronomique de la Chine antique. 2. Cf. également Histoires. Livre II, 158, 17-18, où Hérodote écrit que la mer australe (notiè thalassa) « est la même que celle appelée Érythrée ». L'adjectif notios, signifie « humide » et par extension « du sud » ou « austral », le Notos étant le vent du sud qui apporte la pluie. Nous verrons plus loin que les Grecs et les Romains indiquaient les directions polaires nord/sud par le nom des vents qui soufflent sur ces régions. 
3. Cf. Cheng (1997). Le chapitre 10, "La pensée cosmologique " montre comment les principes antithétiques et complémentaires du yin et du yang alternent cycliquement et, à partir du III ${ }^{\mathrm{e}}$ siècle av. notre ère, vont se combiner avec les cinq éléments ou cinq agents (wuxing), "perçus comme cinq hases ou portions de temps (journée, saison, année, dynastie) correspondant à des qualités déterminées qui se succèdent cycliquement à des points de référence fixés dans l'espace » (Cheng, $1997:$ 256). Ainsi, les couleurs (vert, rouge, jaune, blanc noir) correspondentelles non seulement à une des quatre régions cardinales ou au centre, mais aussi à un agent ou élément en même temps qu'à un nombre : 1 . Eau ; 2. Feu ; 3. Bois ; 4. Métal ; 5. Terre (cf. Granet, 1934/1999: chap. III, IV), qui explique en détail le rôle des nombres dans ce système cosmologique). Entrent encore dans cette classification symbolique quinquénaire toutes sortes de séries de cinq objets : cinq saisons (printemps, été, transition [= centre], automne, hiver); cinq planètes (Mercure, Mars, Jupiter, Venus, Saturne) ; cinq saveurs, cinq vertus, etc. (cf. SunDurand, 2005 : 318-321) qui résume dans un tableau synoptique «la symbolique quinquénaire dans la culture chinoise ".

4. Selon Rémy Dor, que je remercie pour cette communication personnelle, l'association kara/ nord et ak/sud est ancienne et panturque en Asie Centrale.

5. Saussure, frappé par le parallélisme entre la cosmologie indienne et chinoise hésite sur le sens de l'influence qui a pu s'exercer de l'une sur l'autre. Dans son article nécrologique, Gautier (1926 : 38) cite une lettre de Saussure qui dit: « Tout d'abord j'ai incliné à croire le système importé de Chine en Iran, mais j'ai bientôt reconnu le contraire ». Pour Alexander Vovin (communication personnelle, 26/04/2019), la correspondance entre couleurs et orientations cardinales est très ancienne, et certainement antérieure aux civilisations chinoise et védique. Il en donne pour preuve les vestiges qu'on peut en découvrir dans le chamanisme sibérien et dans la pensée des Amérindiens. Ce que confirment Durkheim et Mauss dans leur étude de 1903 (reprise dans Mauss, 1968) : «De quelques formes primitives de classification », ils montrent que le peuple des Zuñis avait édifié un système où l'univers était divisé « en sept régions » (Nord, Sud, Ouest, Est, Zénith, Nadir et Milieu) entre lesquelles «toutes les choses de l'univers sont réparties. [...] À chaque région est attribuée une couleur qui la caractérise. Le Nord est jaune parce que dit-on, au lever et au coucher du soleil la lumière y est jaune. Le Sud est rouge parce que c'est la région de l'été et du feu qui est rouge. L'Est est blanc parce que c'est la couleur du jour. [...] les régions inférieures sont noires comme les profondeurs de la terre » (Mauss, $1968: 192-195)$.

6. La mer Rouge forme un "golfe marin, en Arabie, non loin de l'Égypte, qui de la mer appelée Érythrée, pénètre dans les terres, long et étroit ». (Histoires. Livre II, 11)

7. Il est bien connu que la mer Rouge actuelle ne représente que le golfe le plus occidental de la mer Érythrée, ou « golfe Arabique ». En direction de l'Est, la mer Érythrée, au temps d'Hérodote comprenait un second golfe, le " golfe Persique », notre actuel Golfe Arabo-Persique ou Arabique. Et, elle s'étendait jusqu'au fleuve Indus, couvrant ce que nous appelons aujourd'hui la mer d'Oman. À titre indicatif, quand Hérodote écrit que l'Euphrate (Hist. I, 180) et le Tigre (Hist. I, 189) se jettent dans la mer Érythrée, il désigne le golfe Persique des Grecs, soit notre golfe AraboPersique. La carte du monde habité (œkoumène) selon Strabon ( $\mathrm{au} \mathrm{I}^{\mathrm{er}}$ siècle) dessine un littoral rectiligne, depuis la côte orientale du golfe Persique jusqu'à l'Indus et à l'île de Taprobane (Ceylan). Ce n'est qu'à l'époque impériale romaine, sous le règne des Antonins au II $^{\mathrm{e}}$ siècle de notre ère que l'on découvrira l'Inde au-delà du Gange jusqu'en Chine, avec le commerce de la soie par voie terrestre et maritime (Aujac, 2012 : 119-126). Dans la Géographie de Ptolémée (Livre VII, 5), «La mer de l'Inde» (notre Océan Indien) est décrite comme une mer fermée "entièrement encerclée par la terre ", qui comprend une suite de golfes : " golfe Arabique, golfe Persique, golfe Gangétique et celui qu'on appelle le Grand Golfe » (trad. Aujac, 2012:389). Au vII ${ }^{\mathrm{e}}$ s. encore, on peut lire chez Isidore de Séville : « La mer rouge est divisée en deux golfes. L'un d'eux est appelé golfe Persique parce que les Perses sont établis à son embouchure. L'autre s'appelle Arabique, parce qu'il jouxte l'Arabie » (Étymologies. Livre XIII, 17, 4). 
8. Cf. L'Histoire d'Alexandre le Grand (X, 1, 13-14) de Quinte Curce, où l'historien latin fustige une confusion déjà répandue autour $\mathrm{du} \mathrm{I}^{\mathrm{er}}$ siècle de notre ère. Il rapporte que deux messagers d'Alexandre ont appris des indigènes habitant la région (13) «que la mer Érythrée ne tirait pas son nom, comme c'était l'opinion commune, de la couleur de ses eaux, mais du roi Érythrus ; (14) que, non loin du continent, était une île plantée d'un grand nombre de palmiers, et que, environ au milieu du bois, s'élevait une colonne, monument consacré au roi Érythrus, avec une inscription dans la langue du pays ». (Cf. également Arrien, Indica, 37.3, cité dans Bačić, 1995 : 187) : " They said that in this island [sc. Oaracta, modern Qeshm] the tomb of the first chief of this territory was shown; his name was Erythres, and hence came the name of the sea ».

9. Cf. Strabon, Géographie. Livre XVI, 4, 20 : «Artémidore cite [...] ce qu'Agatharchide - de Cnide dit avoir recueilli de la bouche d'un certain Boxus, originaire de la Perse, au sujet du Perse Erythras, gardien d'un haras. Une lionne, exaspérée par la piqûre d'un taon, avait chassé tous les chevaux jusqu'à la mer et de là jusqu'à une île qu'un bras de mer sépare de la côte. Erythras s'était alors construit un solide radeau, et il avait atteint l'île où aucun homme avant lui n'avait mis le pied. » Séduit par ses richesses, après avoir ramené son troupeau au haras, il revint fonder une colonie dans cette île. Colonie qui s'étendit aux îles voisines et au littoral proche. "Après quoi il avait donné son nom à la mer elle-même ».

10. Cf. Rougé (1988 : 59) : «Si nous prenons pour exemple le Périple de la mer Érythrée, il s'agit de tout l'espace maritime qui s'étend entre l'Afrique et l'Inde au nord, grosso modo, d'une ligne tirée de la région de Zanzibar au cap Cormorin [...]. Pour Pline l'ancien [...] cet espace maritime est «l'océan Indien » et le sinus arabicus est déjà la mer Rouge. [...] Quand les documents d'époque ptolémaïque parlent de l'Érythrée, c'est de la mer Rouge qu'il s'agit ». Voir aussi Schneider (2001).

11. Cf. texte original : «It's widely believed today that the ancient Greeks learned the name Red Sea from the Egyptians who had allegedly transferred the attribute to the adjacent sea from Red Land, the name used by them to designate the Desert of the South as opposed to Egypt proper, called the Black Land».

12. Les quatre vents principaux et les quatre positions cardinales du soleil sont indiqués sur le pourtour des mappemondes, les premiers sous la forme iconique de «souffles" sortant de la bouche de petits visages, les seconds en toutes lettres dans des cartouches. Cette figuration animiste des vents remonte à la mythologie grecque. Chez Homère, Éole est le dieu qui régit les vents (anemoi), en les tenant enfermés dans des «outres». On pourra, par exemple, se reporter aux reproductions $\mathrm{du}$ "monde habité dans la sphère armillaire du $\mathrm{Xv}^{\mathrm{e}} \mathrm{s}$. » dans Aujac (1998: 16-17) ou à « la carte générale » de Nicolas le Germain de 1482 (Ibid. : 18).

13. Par exemple, Hérodote écrit que «les Perses s'étendent jusqu'à la mer du Sud, ou plus exactement la mer du Notos [ou "notienne", votiè thalassa] appelée Érythrée; au dessus d'eux, vers le vent Borée ou "vent du nord" habitent les Mèdes". Et Hérodote continue son énumération, dans la même direction, en la terminant par «les Colchidiens, qui s'étendent jusqu'à la mer septentrionale ». Il emploie en fait l'expression borèiè thalassa « la mer boréenne », (Histoires. Livre IV : 37-40). Puis, Hérodote décrit les « parties de l'Asie » en direction de hespéra « le soir, le couchant ». Enfin, en sens inverse, vers l'Inde, il indique que l'on se dirige " vers l'aurore (eôs) et le soleil levant (helios anatellôn) ». (Hist. IV : 40)

14. Toutefois, d'après la traduction d'Aujac (1998), les légendes des cartes de la Géographie de Ptolémée n'utilisent, en dehors $d u$ "nord », que les positions solaires pour délimiter les régions. Ainsi indique-t-il systématiquement « la carte est limitée : au levant... ; au midi... ; au couchant... ; au nord...".

15. Cf. le lexique spécialisé établi par Germaine Aujac à la fin du tome II de la Géographie de Strabon. À l'entrée anemos (T. II : 179), G. Aujac écrit : « On trouve cités ensemble "les climats et les vents" qui permettent de situer un lieu à la fois par rapport à la latitude ou aux parallèles de la carte (climats), et par rapport à la longitude ou aux méridiens (vents), c'est-à-dire plus 
généralement par rapport aux quatre points cardinaux » (cf. Géogr. I, $2: 20-21$ ). Les quatre vents cardinaux identifiés par un nom de divinité grecque ou romaine occupent les quatre coins des cartes antiques (cf. Aujac, 1988). Chaque vent principal peut être flanqué d'un vent intermédiaire (8 vents), ou encore de deux sur les mappemondes postérieures jusqu'à la Renaissance (Vagnon \& Vallet, 2017 : 32-33). Le catalogue des vents en grec et en latin est donné par plusieurs auteurs (cf. par ex. Météorologiques d'Aristote pour le grec; ou, en latin, le chapitre sur les vents d'Isidore de Séville (liv. XIII, 11) : Est, Subsolanus; Sud, Auster; Ouest, Favonius et le vent qui souffle du Nord porte le même nom Septentrio que la région du nord.

16. Merci à Christoph Habsmaier pour son aide érudite à propos des relations entre points cardinaux et couleurs en chinois. Comme il l'indique, il existe tant de sous-systèmes locaux, qu'il est difficile de dégager un système général.

17. Selon Bačić (1995 : 188), la traduction de la Bible en grec a été un facteur décisif de l'emploi de mer Érythrée pour désigner l'actuelle mer Rouge. Dans la Septante, Eruthra thalassa a servi à traduire l'hébreu Yam suf «mer des joncs / des roseaux », nom de la mer dont Yahvé a écarté les flots pour que son peuple la traverse. Ainsi la mer Érythrée a-t-elle été réduite à la mer de Mertet ou à la Grande Noire des Égyptiens. Un grand merci à P. E. Moog pour sa mise au point sur la façon de nommer (ou le plus souvent de ne pas nommer) les mers dans le texte biblique.

18. Saussure (1924: 31) remarque qu'au XIV ${ }^{e}$ s. les Byzantins utilisaient encore le nom grécoromain de Pont Euxin pour désigner la mer Noire. À la même époque, les géographes et navigateurs italiens la nommaient mer Majeure.

19. Je conserve la transcription en alphabet romain de L. de Saussure, qui s'appuie sur des documents arabes des $\mathrm{X}^{\mathrm{e}}$ et XII ${ }^{\mathrm{e}}$ siècles. (Saussure, $1924: 31$ )

20. Merci à J.-C. Anscombre pour son analyse de la forme a-swad, en un préfixe de comparatif (a-), et la racine /swd/ d'où la traduction de "plus grand » (cf. mar Maiour de Marco Polo). Ce préfixe s'étant lexicalisé avec les adjectifs de couleur, aswad est régulièrement traduit par « noir ».

21. Saussure dit ignorer "à quelle date apparaît l'appellation de mer Blanche » faute de documents écrits. Il relève une occurrence de mer Noire associée à mer Blanche « dans un traité nautique de 1555.» (ibid.: 2, n. 1) Comme l'écrit Bratianu (2009: 34) : «De tous les problèmes concernant la mer Noire, il n'en est pas de plus caractéristique et aussi de moins bien connu que celui de son nom, bien que cette affirmation puisse sembler paradoxale ». Lui-même propose de ramener à une « source commune » iranienne les appellations de Pont Euxin et de mer Noire, en adoptant l'hypothèse selon laquelle euxeinos serait une réfection par euphémisme du grec axeinos « inhospitalier » (cf. Pline l'Ancien, Histoire naturelle. Livre VI, 175 : « une vaste mer, le Pont-Euxin, appelé jadis Axenus»). Il s'agirait d'une adaptation en grec de l'iranien akhshaena. Il mentionne également la double transposition en arabe de Pontus : celle phonétiquement régulière en Buntus et celle déformée en Nitas. Par exemple au $\mathrm{x}^{\mathrm{e}}$ siècle, le géographe abbasside al-Mas'udi, dans Les prairies d'or et mines de gemmes, recense la mer Nitas, Bahr al Nitas parmi les mers de ce globe (https://histoireislamique.files.wordpress.comdroppedimage_1-les-7-mers-dalmasudi.png). Ces mers sont au nombre de 4, 6 ou 7 selon les auteurs. «La mer Nitas, précise-t-il, communique avec la mer Mayotis [lac Méotide chez Hérodote, mer d'Azov aujourd'hui] et se joint à la Méditerranée par le canal de Constantinople ». Ou encore, la mer Nitas figure sur la carte d'Idrisi, au XII ${ }^{\mathrm{e}}$ siècle. Bratianu (ibid. p. 35) émet des réserves sur l'étymologie, a priori déroutante, qui dérive de Pontus les formes Nitas ou Nitash. Merci à Mehdi Ghouirgate pour avoir clarifié cette transposition qui, selon lui, s'explique à la fois phonétiquement et graphiquement. Car, me précise Osman Karatay, le déchiffrage des manuscrits arabes médiévaux entraîne des confusions entre les consonnes de forme voisine $\mathrm{B}$ (transcription de $\mathrm{P}$ ) et $\mathrm{N}$, qui se distinguent par un point suscrit ou souscrit pas toujours lisible. Quant aux voyelles non notées, elles sont lues de manière fantaisiste dans les noms propres inconnus. O. Karatay cite par exemple Bulgare transcrit Nilgaz. Cf. aussi, l'article Kara Deniz, dans First encyclopédia of Islam (1913-1936) de Brill (1993) et Gordeev (2007), Cartography of Black and Azov Seas: Retrospective up to 1700. 
22. Cf. la description que Pline l'Ancien donne du royaume des Perses "placé entre deux mers, celle de Perse et celle d'Hyrcanie » (Hist. Nat. Livre VI : 16), repris quelques chapitres plus loin avec une variante: «les royaumes situés le long de deux mers, la mer Rouge au midi, la mer Hyrcanienne au nord » (Hist. Nat. Livre VI : 29) en remplaçant la mer de Perse par la mer Rouge.

23. Je dois ces précisions à Osman Karathay que je remercie vivement pour tous les éclaircissements qu'il a eu l'amabilité de m'apporter, en réponse à mes (trop) nombreuses questions.

24. Il est intéressant de noter que l'OHI n'a fait que systématiser les deux façons de nommer les mers qui étaient les plus courantes dans l'antiquité, selon le témoignage d'Isidore de Séville (Etym. Livre XIII, 16:5-7). Après avoir remarqué que "de même qu'un seul lieu est nommé différemment selon les endroits, de même "la Grande mer Extérieure" reçoit différents noms selon les régions ». Ces noms, précise-t-il, sont ceux des « provinces » qui la bordent; ou des îles qui s'y situent; ou encore des peuples riverains. On reconnaît ici le groupe dominant des toponymes. Isidore mentionne également la possibilité de nommer une mer en mémoire d'un roi (Mer Ionienne, d'après le roi Io) qui se rapproche du second groupe, et d'autres disparues aujourd'hui.

\section{RÉSUMÉS}

Le nom turc de la Méditerranée est Akdeniz, ou mer Blanche, qui s'oppose à Karadeniz, ou mer Noire. Cet exposé présente et critique le principe explicatif selon lequel la couleur dans le nom des mers ne correspond pas à une propriété de leurs eaux, mais indique la localisation géographique de ces mers, par référence à la couleur attachée aux points cardinaux dans différentes cultures.

The Turkish name for the Mediterranean Sea is Akdeniz, that is "White Sea", as opposed to Karadeniz or "Black Sea". This study aims at examining and criticizing the explanatory principle according to which the colour in such sea-names does not fit with some natural property of their waters, but rather points out to the geographical localization of those seas by referring to the specific colours attached to the cardinal points in several cultures.

\section{INDEX}

Mots-clés : couleurs géographiques, points cardinaux, systèmes de dénomination des mers

Keywords : geographical colours, cardinal points, sea naming system

\section{AUTEUR}

\section{IRÈNE TAMBA}

CRLAO (Centre de recherches linguistiques sur l'Asie orientale), EHESS-Paris 